\title{
CONSUMO DE EDULCORANTES ARTIFICIAIS POR GESTANTES E NUTRIZES DO TOCANTINS
}

Consumption of artificial sweeteners by pregnant women and nutrites in Tocantins

Consumo de edulcorantes artificiales por mujeres embarazadas y nutritos em Tocantins

\section{Priscila de Souza Barros ${ }^{1}$, Tatiana Vieira Ferreira ${ }^{2}$, Renata Junqueira}

\section{Pereira*3}

${ }^{1}$ Discente do Curso de Nutrição, Universidade Federal do Tocantins, Palmas, Brasil.

${ }^{2}$ Mestranda em Ciências da Saúde, Universidade Federal do Tocantins, Palmas, Brasil.

${ }^{3}$ Professora Doutora do Curso de Nutrição, Universidade Federal do Tocantins, Palmas, Brasil.

*Correspondência: Universidade Federal do Tocantins, Quadra 109 Norte, avenida NS15, ALCNO 14, LACIBS,

Estação experimental. Campus Universitário de Palmas. E-mail:renatajunqueira@ mail.uft.edu.br

\section{Artigo recebido em 23/06/2021 aprovado em 26/10/2021 publicado em 18/11/2021}

\section{RESUMO}

O cenário atual da alimentação humana indica um aumento crescente do consumo de produtos industrializados e ultraprocessados, bem como produtos artificialmente adoçados que, inicialmente formulados para suprir às necessidades da população diabética, hoje atendem a tendência da população em reduzir e controlar o consumo de calorias. Apesar de consideradas seguras pelas autoridades de saúde, são contraindicadas na gestação e lactação. Com o objetivo de conhecer a utilização e quantificar o consumo diário total de edulcorantes artificiais por gestantes e lactantes, foi realizada a coleta de dados sobre o consumo alimentar, avaliando quais produtos continham edulcorantes em sua composição e seus, respectivos teores diante do consumo diário individual e análise com a ingestão diária aceitável (IDA). Dos 161 recordatórios coletados, 89 eram gestantes e 72 nutrizes, sendo possível quantificar o consumo de edulcorantes por 9 das gestantes e 15 das nutrizes. Não foi encontrado consumo acima da ingestão diária aceitável para nenhuma das participantes. Em face do exposto, ressalta-se a importância do acompanhamento nutricional com orientações e recomendações conscientes.

Palavras-chave: adoçantes artificiais; gestação; lactação.

\section{ABSTRACT}

The current scenario of human food indicates an increasing increase in the consumption of industrialized and ultra-processed products, as well as artificially sweetened products that, initially formulated to meet the needs of the diabetic population, today meet the population's tendency to reduce and control the consumption of calories. Despite being considered safe by health authorities, they are contraindicated during pregnancy and lactation. In order to know the use and quantify the total daily consumption of artificial sweeteners by pregnant women and lactating mothers, data were collected on food consumption, evaluating which products contained sweeteners in their composition and their respective levels in relation to individual daily consumption. and analysis with acceptable daily intake (ADI). Of the 161 food records collected, 89 were pregnant women and 72 nursing mothers, and it was possible to quantify the consumption of sweeteners by 9 of the pregnant women and 15 of the nursing mothers. No consumption was found above the acceptable daily intake for any of the participants. In view of the above, the importance of nutritional monitoring with conscious guidance and recommendations is emphasized.

Keywords: artificial sweeteners; gestation; lactation. 


\section{RESUMEN}

El escenario actual de la alimentación humana indica un consumo creciente de productos industrializados y ultraprocesados, así como de productos endulzados artificialmente que, inicialmente formulados para satisfacer las necesidades de la población diabética, hoy responden a la tendencia de la población a reducir y controlar el consumo de calorías. . Aunque las autoridades sanitarias las consideran seguras, están contraindicadas en el embarazo y la lactancia. Para conocer el uso y cuantificar el consumo diario total de edulcorantes artificiales por parte de mujeres embarazadas y lactantes, se recogieron datos sobre el consumo de alimentos, evaluando qué productos contenían edulcorantes en su composición y sus respectivos niveles en relación al consumo diario individual y análisis con ingesta diaria aceptable (IDA). De los 161 retiros recogidos, 89 eran embarazadas y 72 lactantes, y fue posible cuantificar el consumo de edulcorantes por 9 de las embarazadas y 15 de las lactantes. No se encontró un consumo por encima de la ingesta diaria aceptable para ninguno de los participantes. En vista de lo anterior, se destaca la importancia del seguimiento nutricional con orientación y recomendaciones conscientes.

Descriptores: edulcorantes artificiales; gestación; lactancia

\section{INTRODUÇÃO}

Os adoçantes ou edulcorantes, são substâncias químicas, obtidas de matéria primas naturais ou artificiais, desenvolvidas pela indústria de alimentos, com o poder de substituir total ou parcialmente o açúcar, sem acrescentar calorias à preparação (GONZALES et al., 2016).

Inicialmente formulados para atender às necessidades da população diabética, os adoçantes nos dias atuais também são utilizados em processos de emagrecimento e, em decorrência da epidemia de obesidade, vêm sendo cada vez mais utilizados como forma de reduzir e controlar o consumo de calorias pela população. Condições como o culto ao corpo e à saúde contribuem para a crescente utilização e comercialização desses produtos (TORLONI et al., 2007).

Considerados substâncias eficazes para adoçar, em pequenas concentrações, os edulcorantes atualmente passaram a ser comercializados em fórmulas, contendo dois ou mais tipos de substâncias, para potencializar as vantagens de cada tipo e neutralizar o sabor residual (SAUNDERS et al., 2010).

Apesar de serem considerados seguros pelas autoridades de saúde, o uso de edulcorantes divide a comunidade científica, uma vez que recebem uma classificação de risco potencial para uso na gravidez, pelo Food and Drug Administration (FDA, 1980), sendo divididos em grupos: A - estudos controlados em mulheres não demonstraram risco para o feto no primeiro trimestre; não existe evidência de risco nos outros trimestres e a possibilidade de dano fetal parece ser remota; B - estudos em animais não indicam risco fetal e não há estudos controlados na espécie humana ou, ainda, estudos em animais demonstram efeito adverso no feto, mas estudos bem controlados em mulheres grávidas não demonstraram risco para o feto; $\mathrm{C}$ - estudos têm mostrado que as substâncias apresentam efeito teratogênico ou embriocida em animais, mas não há estudos controlados em mulheres, ou ainda não há estudos controlados nem em animais e nem em mulheres. Essas substâncias só devem ser administradas se os possíveis benefícios justificarem os riscos potenciais para o feto; D - existem evidências de risco ao feto humano, mas os benefícios, em certas situações (risco de morte ou doenças graves, para as quais substâncias mais seguras são ineficazes ou não podem ser usadas), podem fazer com que o uso dessa substância seja aceitável, apesar do seu risco; X - estudos em animais ou humanos têm demonstrado anormalidades fetais ou há evidências de risco fetal baseadas em estudos em humanos, ou ambos, e os riscos 
associados ao uso da substância na gestação claramente superam quaisquer benefícios possíveis. Essas substâncias são contraindicadas em mulheres grávidas ou que podem vir a engravidar.

Atualmente existem 7 tipos de adoçantes liberados no Brasil pela Agência Nacional de Vigilância Sanitária (ANVISA) e suas Ingestões Diárias Aceitáveis (IDA), em $\mathrm{mg} / \mathrm{kg} / \mathrm{dia}$, foram estabelecidas pela Organização Mundial de Saúde (WHO, 2017). A sacarina sódica e o ciclamato de sódio se enquadram na categoria de risco $\mathrm{C}$ e possuem ingestão diária aceitável (IDA) de 5 e 11 $\mathrm{mg} / \mathrm{Kg}$ peso/dia, respectivamente. $\mathrm{O}$ acessulfame de potássio e a sucralose estão enquadrados na categoria B e possuem IDA de 15 e $5 \mathrm{mg} / \mathrm{Kg}$ peso/dia. O aspartame possui IDA de $40 \mathrm{mg} / \mathrm{kg}$ peso/dia e quanto ao uso por nutrizes, recomenda-se $34 \mathrm{mg} / \mathrm{kg}$ peso/dia. Em 2008 as substâncias neotame e taumatina foram permitidas no Brasil, sendo a IDA para o neotame de $2 \mathrm{mg} / \mathrm{Kg}$ peso/dia. A substância taumatina não possui ainda limite de ingestão diária estabelecida (SAUNDERS et al., 2010).

Segundo Natividade (2011), a IDA é calculada para a população em geral, ao passo que a gestação é uma situação peculiar e exige estudos específicos a respeito da segurança de seu uso. A utilização da sacaria sódica, do aspartame e do ciclamato de sódio deve ser evitada na gestação, dado seu potencial carcinogênico em experiências com animais e a escassez de estudos disponíveis acerca de seus efeitos sobre a gestante e o feto.

Diante disso, durante a gravidez e lactação o uso de adoçantes deve ser reservado para as pacientes que necessitam de controle do ganho de peso e para as diagnosticadas com diabetes gestacional, com ressalva de consumo moderado. $\mathrm{O}$ estado nutricional adequado de uma gestante, resultante da associação alimentação equilibrada e ganho de peso dentro das faixas de normalidade, pode contribuir para a diminuição da morbimortalidade materno-infantil (PADILHA et al., 2010).

Dessa forma, faz-se importante conhecer a utilização e quantificar o consumo diário total de edulcorantes, bem como seus alimentos fontes na dieta de gestantes e lactantes, considerando-se a vulnerabilidade deste grupo ao risco teratogênico e aos contaminantes do leite materno.

\section{MATERIAIS E MÉTODOS}

As equipes de Estratégia Saúde da Família (ESF) de Palmas, foram consideradas como organização da distribuição territorial da população a ser examinada.

A seleção das gestantes e nutrizes a serem investigadas em cada Unidade Básica de Saúde UBS foi realizada da lista completa de todas as gestantes e nutrizes acompanhadas mensalmente na unidade, durante todo o período de vigência da pesquisa.

Em seguida houve o sorteio de 100 gestantes e 100 lactantes que fariam parte do estudo. Devido à suspensão do estudo em março de 2020, por causa da pandemia de Covid-19, foram entrevistadas 91 gestantes e 65 nutrizes.

De todas elas foram coletados os dados de inquérito dietético, através da aplicação de um recordatório alimentar de 24 horas, como forma de avaliar o consumo alimentar das últimas 24 horas, detalhando informações quali e quantitativas, como o tipo de alimento consumido, marcado produto utilizado, o horário, as refeições em que foram consumidas, o local do consumo, o modo de preparo, as porções consumidas e suas respectivas quantidades. 
Os produtos obtidos na coleta de dados sobre consumo alimentar tiveram suas informações conferidas no lote da marca ou nome comercial, para avaliar quais os edulcorantes os compunham e os seus respectivos teores.

Para o cálculo desses teores foi usada a quantidade diária de alimento contendo edulcorantes, consumida pela paciente, bem como o tipo de edulcorante e sua classe química.

A ingestão da paciente em relação à ingestão diária aceitável (IDA) foi avaliada conforme proposto por Saunders (2010), onde sacarina sódica possui IDA de $5 \mathrm{mg} / \mathrm{kg}$ peso/dia, ciclamato de sódio de 11 $\mathrm{mg} / \mathrm{kg}$ peso/dia, acessulfame de potássio de $15 \mathrm{mg} / \mathrm{kg}$ peso/dia e o aspartame de $40 \mathrm{mg} / \mathrm{kg}$ peso/dia, quanto ao uso por nutrizes, recomenda-se $34 \mathrm{mg} / \mathrm{kg}$ peso/dia. Padronizou-se utilizar o peso atual das gestantes e, para as nutrizes, foi considerado o último peso gestacional, obtido após as 36 semanas, descontando $7 \mathrm{Kg}$ desse peso, correspondentes ao bebê e anexos embrionários. Para as mulheres cuja informação não estava disponível, considerou-se o peso padrão para mulheres adultas, de $60 \mathrm{Kg}$, proposto pelas Dietary Reference Intakes (IOM, 2005).

Os dados foram tabulados e os cálculos realizados no Microsoft Excel 365 e as análises estatísticas descritivas, no Statistical Package for Social Sciences (SPSS), versão 20.0.

O protocolo do presente estudo foi aprovado pelo Comitê de Ética em Pesqusias com Seres Humanos da UFT, sob número de parecer 2.702.535 e as participantes assinam em duas vias o termo de consentimento livre e esclarecido.

\section{RESULTADOS E DISCUSSÃO}

Foram coletados 161 recordatórios, sendo 89 de gestantes e 72 nutrizes. Do total de gestantes entrevistadas, apenas 16 referiam consumir edulcorantes em seus recordatórios e das 72 nutrizes, 19 referiam o consumo dessas substâncias.

Foi possível quantificar o consumo de edulcorantes artificiais para 9 gestantes e 15 nutrizes. Diante desses números, optou-se por uma análise quali-quantitativa.

O acessulfame de potássio e o aspartame foram os edulcorantes mais utilizados no grupo das nutrizes, sendo citados 26 vezes, em alguns casos com ingestão superior a três vezes ao dia. Os outros edulcorantes encontrados neste grupo foram $o$ ciclamato e a sacarina, referidos 24 vezes na análise, em alguns casos com ingestão superior a duas vezes ao dia. Entre as gestantes não houve consumo elevado de um edulcorante específico, sendo o aspartame, ciclamato, acessulfame e sacarina referidos 11 vezes nos recordatórios analisados.

Comparando os resultados obtidos com estudos recentes em relação à segurança no uso dos edulcorantes, temos uma recomendação de cautela quanto ao uso da sacarina devido à falta de informações conclusivas sobre possíveis efeitos no desenvolvimento fetal (SAUNDERS, 2010). Conforme Freitas (2005) a sacarina possui absorção lenta e incompleta e, apesar de apresentar completa eliminação renal, gestantes devem restringir o uso devido à permeabilidade placentária à sacarina, que pode permanecer nos tecidos fetais devido a menor capacidade do feto em excretá-la.

O ciclamato, produto sintético, também apresenta capacidade de atravessar a placenta, podendo causar efeitos citogenéticos sobre os linfócitos humanos, associando-se ao aumento da incidência de malformações e problemas comportamentais nos fetos das gestantes que consumiram (VITOLO, 2003). 
Rapidamente absorvido e com excelente estabilidade, o acessulfame não é metabolizado e o organismo elimina pela urina (FREITAS, 2005). Apesar de aparecer no leite materno durante o período da lactação, não foi observado efeito do acessulfame sobre o bebê, nem efeito mutagênico ou teratogênico quando utilizado por gestantes (SAUNDERS, 2010).

Quanto ao aspartame, de acordo com Saunders (2010), não há evidência comprovando efeitos negativos na lactação, nem quanto a sua capacidade de atravessar a placenta. Seu uso deve ser evitado em mulheres com perfil positivo para fenilcetonúria, uma vez que a concentração sérica fetal de fenilalanina é cerca de duas vezes maior que a encontrada no cordão umbilical. $\mathrm{O}$ aumento dos níveis de fenilalanina associado com a ingestão de aspartame em quantidade normalmente utilizada pela população pode ocasionar variações no quociente de inteligência (QI), refletindo no desenvolvimento fetal.

Portanto, segundo as evidências atualmente disponíveis, e considerando as gestantes com preocupação quanto ao ganho de peso excessivo e as nutrizes em processo de recuperação do peso prégestacional, o aspartame, a sucralose, o acessulfame e a estévia podem ser utilizados com segurança durante a gestação (TORLONI et al., 2007).

Em relação às principais fontes de edulcorantes das dietas analisadas observamos o consumo de refrigerantes, sucos artificiais e gelatinas, alimentos estes com baixo valor nutricional, pobres em proteínas, vitaminas e minerais, alta quantidade de corantes e substâncias químicas que não deveriam ser consumidas na gestação, tendo em vista a importância da relação alimentação adequada e gestação, período de maior demanda nutricional do ciclo de vida da mulher (CASTRO; FRANCO, 2002).

A análise do consumo diário de edulcorantes em relação à Ingestão Diária Admissível (IDA) revelou que nenhuma das gestantes ou nutrizes consumiam edulcorantes além do limite máximo diário aceitável.

Os teores médios diários de edulcorantes consumidos pelas participantes desse estudo foram $84,6 \mathrm{mg} / \mathrm{dia}$ de aspartame, $66,1 \mathrm{mg} / \mathrm{dia}$ de ciclamato, $13,2 \mathrm{mg} / \mathrm{dia}$ de acessulfame e $13,8 \mathrm{mg} / \mathrm{dia}$ de sacarina, pelas gestantes. No caso das nutrizes, o consumo médio diário foi de $114,07 \mathrm{mg} / \mathrm{dia}$ de aspartame, $82,2 \mathrm{mg} /$ dia de ciclamato, $19,28 \mathrm{mg} / \mathrm{dia}$ de acessulfame e 9,67 mg/dia de sacarina.

\section{CONCLUSÃO}

Diante do exposto, durante a gestação e lactação, apesar da grande disponibilidade no mercado, a escolha do adoçante deve ser cuidadosa, pois determinadas substâncias utilizadas em sua formulação podem ser prejudiciais neste período e mesmo as citadas como seguras, não há evidências científicas concretas.

Ressalta-se a importância dos serviços de saúde, quanto ao monitoramento do pré-natal e acompanhamento puerperal, orientando as mulheres de forma adequada, para que as recomendações nutricionais possam incentivar uma melhoria na qualidade da alimentação e, consequentemente, promover saúde para o binômio mãe-filho.

\section{AGRADECIMENTO}

O presente trabalho foi realizado com o apoio do Conselho Nacional de Desenvolvimento Científico e Tecnológico - CNPq - Brasil, ao qual agradecemos o auxílio na execução deste projeto. 
Todos os autores declararam não haver qualquer potencial conflito de interesses referente a este artigo.

\section{REFERÊNCIAS}

CASTRO, A.; FRANCO, L. Caracterização do consume de adoçantes alternativos e produtos dietéticos por indivíduo diabéticos. Arquivo Brasileiro de Endocrinologia e Metabologia.Vol.43. 2002.

FOOD AND DRUG ADMINISTRATION (FDA). Pregnancy categories. Fed Regist [serial on the Internet]. 1980. Disponível em: <http://www.medicalcorps.org/pharmacy/Pregnancy Categories.htm>. Acesso em: 14 mar. 2019.

FREITAS, S.M.L. Alimentos com Alegação Diet ou Light: Definições, Legislação e Implicações no Consumo. 1. ed. São Paulo: Atheneu, 2005. 138 p.

GONZALES C. R., SILVA G. B., FERRO E. L. B. S. Adoçantes recomendados para gestantes diabéticas. In: $16^{\circ}$ Congresso Nacional de Iniciação Científica. CONIC - SEMESP- Universidade de Franca. 2016, Guarulhos, São Paulo. Anais eletrônicos. [acesso em 07 mar 2019]. Disponível em: http://conic-semesp.org.br/anais/files/2016/trabalho1000023122.pdf

INSTITUTE OF MEDICINE (IOM). Dietary reference intakes for energy, carbohydrate, fiber, fat, fatty acids, cholesterol, protein, and amino acids. National Academic Press: Washington, DC, USA, 2005. Disponível em: < https://www.nap.edu/catalog/10490.html >. Acesso em 14 mai. 2019
NATIVIDADE, D.P. Uso de adoçantes dietéticos: orientações para profissionais de saúde e de ensino. [Dissertação]. Fundação Oswaldo Aranha, Centro Universitário de Volta Redonda. Mestrado profissional em ensino em ciências da saúde e do meio ambiente. 2011. $64 \mathrm{p}$.

PADILHA, P. C. et al. Terapia nutricional no diabetes gestacional. Revista de Nutrição, v. 23, n. 1, p. 95-105, 2010.

SAUNDERS, C.; PADILHA, P. C.; LIMA, H. T.; OLIVEIRA, L. M.; QUEIROZ, J. A.; THEME, M. L. M. Revisão da literatura sobre recomendações de utilização de edulcorantes em gestantes portadoras de diabetes mellitus. Femina, v. 38, n. 4, p.179-184, 2010.

TORLONI, M. R. et al. O uso de adoçantes na gravidez: uma análise dos produtos disponíveis no Brasil. Revista Brasileira de Ginecologia e Obstetrícia, Rio de Janeiro, v. 29, n. 5, maio 2007.

VITOLO, M.R. Nutrição: da gestação à adolescência. Rio de janeiro: Richmann\& Autores editores, p. 315, 2003.

WORLD HEALTH ORGANIZATION (WHO). Evaluations of the Joint FAO/ WHO Expert Committee on Food Additives (JECFA). Disponível em: $<$ http://apps.who.int/food-additivescontaminants-jecfa-

database/chemical.aspx?chemID=267>. Acesso em 14 mai. 2019. 